\title{
Avaliação do grau de corrosão de fios ortodônticos submetidos a desafio de pH
}

\author{
Evaluation of the corrosion degree of orthodontic wires subjected to $\mathrm{pH}$ challenge
}

\author{
Kalil Ayres Santana ${ }^{1}$, Danilo Barral de Araujo ${ }^{2 *}$
}

${ }^{1}$ Acadêmico do Curso de Odontologia da Universidade Federal da Bahia (UFBA); Professor Doutor Associado de Bioquímica Oral e do Programa de Pós-graduação em Processos Interativos dos Órgãos e Sistemas da UFBA.

\begin{abstract}
Resumo
Introdução: a movimentação dentária requer a aplicação de um sistema de forças que é orientado com o uso de acessórios, incluindo fios ortodônticos. Existem diversos materiais e fabricantes disponíveis no mercado, por isso é importante que o profissional conheça a composição deste material, uma vez que estará em contato com a mucosa oral, podendo sofrer corrosão e liberação de íons metálicos. Objetivo: avaliar, in vitro, o grau de corrosão de duas ligas metálicas que compõem fios ortodônticos de três fabricantes diferentes, submetidos à ciclagem de pH. Metodologia: 60 corpos de prova foram confeccionados com dois tipos de ligas metálicas ( $\mathrm{NiTi}$ e $\mathrm{CrNi}$ aço 302) produzidas por três fabricantes diferentes, divididos em 6 Grupos de Testes com 10 unidades cada. Os corpos-de-prova foram submetidos à ciclagem ácida de $\mathrm{pH} 4,3$, diariamente, e mantidos em estufa a $37^{\circ} \mathrm{C}$ por 14 dias. A massa foi pesada antes e após a ciclagem, por meio de balança analítica de precisão. A avaliação das características visuais dos fios foi realizada por um único observador em lupa estereomicroscópica. Resultados: a ciclagem de pH dos fios ortodônticos não promoveu perda significativa de massa ou corrosão, apesar da análise das características visuais terem mudado após a ciclagem. Conclusão: com base nos resultados do protocolo experimental estabelecido no presente estudo, a análise do comportamento das ligas de aço $\mathrm{NiTi}$ e $\mathrm{CrNi}-302$ que compõem os fios ortodônticos,

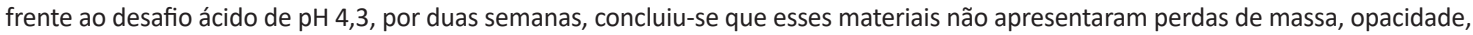
brilho e lisura consideradas significativas. Outros estudos são recomendados para fornecer maiores esclarecimentos sobre o tema.
\end{abstract} Palavras-chave: Ortodontia. Corrosão. Fios ortodônticos.

\begin{abstract}
Introduction: the tooth movement requires the application of a force system that is guided with the use of accessories, including orthodontic wires. There are several materials and manufacturers available on the market, so it is important that the professional knows the composition of this material, since it will be in contact with the oral mucosa, and may suffer corrosion and release metal ions. Objective: to evaluate, in vitro, the degree of corrosion of two metal alloys that make up orthodontic wires from three different manufacturers, submitted to $\mathrm{pH}$ cycling. Methodology: 60 specimens were made from two types of metal alloys (NiTi and CrNi-steel 302) produced by three different manufacturers, divided into 6 Test Groups with 10 units each. The specimens were subjected to acid $\mathrm{pH} 4.3 \mathrm{cycling}$, daily, and kept in an oven at $37^{\circ} \mathrm{C}$ for 14 days. The mass was obtained before and after cycling, using a precision analytical balance. The evaluation of the visual characteristics of the wires was performed by a single observer using a stereomicroscopic loupe. Results: the pH cycling of orthodontic wires did not promote significant loss of mass or corrosion, despite the analysis of visual characteristics having changed after cycling. Conclusion: based on the results of the experimental protocol established in the present study, the analysis of the behavior of the NiTi and CrNi-302 steel alloys that make up the orthodontic wires, facing the acid challenge of $\mathrm{pH} 4.3$, for two weeks, it was concluded that these materials did not present loss of mass, opacity, brightness and smoothness considered significant. Other studies are recommended to provide further clarification on the topic.

Keywords: Orthodontics. Corrosion. Orthodontic Wires.
\end{abstract}

\section{INTRODUÇÃO}

Os fios ortodônticos são peças fundamentais dos aparelhos dentários uma vez que asseguram a aplicação das forças de movimentação que são capazes de gerar a funcionalidade requerida pelo tratamento. No mercado brasileiro existe uma grande variedade de materiais, além de uma vasta gama de fabricantes. É essencial, portanto, que os profissionais tenham o conhecimento adequado sobre a composição, o mecanismo de ação e, até mesmo, sobre as propriedades dos diferentes tipos de ligas metá-

Correspondente/Corresponding: *Danilo Barral de Araújo - Departamento de Bioquímica e Biofísica. Instituto de Ciências da Saúde. UFBA - End: Av. Reitor Miguel Calmon S/N. Vale do Canela. Salvador, BA CEP: 40.110-100 - Tel: (71) 98737-1686 - E-mail: Danilobarral81@ hotmail.com licas que podem vir a compor os fios ${ }^{1}$. Faz-se importante conhecer, ainda, a interação entre estes materiais e os fluidos orais - saliva - uma vez que os mesmos apresentam variação de $\mathrm{pH}$, podendo acarretar em subsequente perda de substância - oxidação - por parte dos fios e exposição dos tecidos moles bucais a tais elementos ${ }^{2}$.

Dentre as ligas metálicas mais aplicadas na ortodontia estão as do tipo níquel-titânio (NiTi), aço inoxidável (SS), beta-titânio e variações de NiTi que são revestidas de cobre (CuNiTi), além de outros tipos ${ }^{1}$. Os componentes dos fios ortodônticos ficam em constante contato com a cavidade bucal podendo sofrer alterações bioquímicas oriundas da ação da saliva e dos alimentos. Nesse contexto, existe a possibilidade da ocorrência de corrosão dessas ligas metálicas devido ao fato do desgaste inicial ser promovido pelos agentes internos, dentre eles a pró- 
pria saliva que é composta por eletrólitos como o sódio, o potássio, o cloreto e o bicarbonato, por exemplo, além dos componentes orgânicos como as enzimas, imunoglobulinas, glicoproteínas e albuminas ${ }^{3}$, reconhecidamente substratos para os microrganismos presentes. Há ainda o contato com agentes externos como os alimentos e bebidas como, por exemplo, os refrigerantes ricos em acidulantes como ácido fosfórico, ácido cítrico e o ácido tartárico, capazes de provocar a corrosão das ligas².

A resistência à corrosão é um fator fundamental na escolha de um fio para o tratamento ortodôntico, isso porque a corrosão pode afetar a lisura do fio e com isso aumentar $o$ atrito entre ele e o braquete, diminuindo em consequência a vida útil, além da possibilidade de manchar tecidos moles ${ }^{4}$. Dentre as formas de corrosão do metal existe a corrosão galvânica e a corrosão eletroquímica ${ }^{2,5}$. A corrosão galvânica ocorre quando dois metais diferentes entram com contato podendo resultar em polarização, enquanto que a corrosão eletroquímica é um processo espontâneo que ocorre quando o metal está presente num meio aquoso, a exemplo do ambiente bucal devido à presença de grande quantidade de eletrólitos que são capazes de gerar reações anódicas e catódicas ${ }^{6}$.

A introdução das ligas de níquel-titânio, em 1971, foi acompanhada por importantes avanços nos tratamentos ortodônticos ${ }^{7}$. Apesar das pesquisas demonstrarem que o titânio é um material biocompatível, sendo inclusive utilizado amplamente pela odontologia nos implantes, a exposição à presença do níquel deve ser cautelosa ${ }^{8}$, uma vez que existem registros na literatura científica que relacionam este íon a possíveis reações alérgicas. O potencial dos fios ortodônticos em causar reações alérgicas está relacionado ao padrão e ao modo de corrosão, com a subsequente liberação de íons metálicos, a exemplo do níquel no meio bucal ${ }^{9}$.

A cavidade bucal sofre com alterações de $\mathrm{pH}$, além de fenômenos enzimáticos e microbiológicos que podem causar alguma interferência nas ligas presentes nos fios ortodônticos, implicando numa aceleração do processo corrosivo. Essa informação é relevante tanto para o ortodontista quanto para o paciente, pois os problemas de maloclusão dentária geralmente demandam um tempo considerável de tratamento e, consequentemente, os fios que compõem o aparelho vão estar em contato com a mucosa por um período de tempo relevante. Logo, os fios ortodônticos devem ser resistentes à corrosão, não devem permitir a liberação de íons na cavidade bucal, tampouco devem ser capazes de gerar alergias, assim sendo, esses materiais devem efetivamente ser biocompatíveis ${ }^{10}$.

Considerando a importância deste tema para a formulação do plano de tratamento e visando auxiliar os especialistas na escolha do fio mais adequado para cada etapa da mecânica ortodôntica, haja visto o potencial corrosivo que cada metal possui, buscou-se através deste estudo avaliar o grau de corrosão de duas ligas metálicas procedentes de três diferentes fabricantes, amplamente utilizadas em fios ortodônticos comercializados no mercado nacional, frente ao teste laboratorial de ciclagem de $\mathrm{pH}$.

\section{METODOLOGIA}

\section{SELEÇÃO E PREPARO DOS CORPOS-DE-PROVAS}

Para a realização deste estudo foram confeccionados corpos-de-prova a partir da utilização de dois tipos de ligas metálicas ( $\mathrm{NiTi}$ e $\mathrm{NiCr}$ - aço 302) procedentes de três fabricantes distintos (Morelli - Dental Morelli Ltda, SP, Brasil; Alditek do Brasil Ltda, SP, Brasil; Orthometric - Indústria e Comércio de Produtos Médicos e Odontológicos, SP, Brasil). Inicialmente, os fios ortodônticos foram cortados com auxílio de alicates de corte, instrumentos próprios da ortodontia, sendo utilizada uma régua flexível, lápis dermatográfico, luvas de látex e pinça de plástico para não haver contato com o suor devido à presença de sais e íons que podem promover a corrosão. Diante da grande variedade de fios ortodônticos quanto à espessura e ao padrão geométrico, foi escolhido para a realização desta pesquisa o fio retangular de 017' por $025^{\prime}$. O preparo foi finalizado após a imersão dos espécimes em Acetona Pró-análise Merck S.A. durante dois minutos a fim de remover as impurezas, seguindo-se de secagem à temperatura ambiente por 24 horas, em uma placa de petri. Concluído este processo, um único observador selecionou para a experimentação apenas as amostras que apresentaram brilho uniforme, ausência de ranhuras e fissuras que impossibilitassem a realização das observações com o auxílio da Lupa Estereomicroscópio de Luz Opton (modelo TNE - 10b binocular), obtendo-se ao final, 60 unidades $(20 \mathrm{~mm} \mathrm{x}$ $0,43 \mathrm{~mm} \times 0,63 \mathrm{~mm})^{4}$.

\section{GRUPOS EXPERIMENTAIS}

Foram utilizados 60 corpos-de-prova divididos aleatoriamente em seis grupos testes, totalizando dez espécimes por grupo, como está explicitado na Tabela 1.

Tabela 1 - Divisão dos grupos de estudo de acordo os tipos de liga metálica e fabricantes.

\begin{tabular}{lll}
\hline \multicolumn{1}{c}{$\begin{array}{c}\text { GRUPO } \\
\text { EXPERIMENTAL }\end{array}$} & TIPO DE LIGA & \multicolumn{1}{c}{ FABRICANTE } \\
GT1 & $\mathrm{CrNi}-$ aço 302 & $\begin{array}{l}\text { Morelli (Dental Morelli Ltda, } \\
\text { SP, Brasil) }\end{array}$ \\
\hline GT2 & $\mathrm{NiTi}$ & $\begin{array}{l}\text { Morelli (Dental Morelli Ltda, } \\
\text { SP, Brasil) }\end{array}$ \\
\hline GT3 & $\mathrm{CrNi}-$ aço 302 & Alditek do Brasil LTDA, SP, Brasil \\
GT4 & $\mathrm{NiTi}$ & $\begin{array}{l}\text { Alditek do Brasil LTDA, SP, Brasil } \\
\text { GT5 }\end{array}$ \\
CrNi-aço 302 & $\begin{array}{l}\text { Orthometric - Indústria e Co- } \\
\text { mércio de Produtos Médicos e } \\
\text { Odontológicos, SP, Brasil }\end{array}$ \\
\hline GT6 & $\mathrm{NiTi}$ & $\begin{array}{l}\text { Orthometric - Indústria e Co- } \\
\text { mércio de Produtos Médicos e } \\
\text { Odontológicos, SP, Brasil }\end{array}$ \\
\hline
\end{tabular}

Fonte; Dados da pesquisa 


\section{CICLAGEM E AVALIAÇÃO DA MASSA}

Para realizar a ciclagem foi utilizada a saliva artificial com o pH ajustado a 4.3 e 7.0, respectivamente. Solução A: saliva artificial desmineralizante (DES) preparada com $40 \mathrm{~mL}$ de tampão ácido contendo $2,0 \mathrm{mmol} / \mathrm{L} \mathrm{Ca}^{+2}, 2,0$ $\mathrm{mmol} / \mathrm{L}\left(\mathrm{PO}^{-3}\right)_{4}$ e 0,075 acetato de $\mathrm{mol} \mathrm{em} \mathrm{pH} \mathrm{4.3;} \mathrm{Solução}$ $B$ : saliva artificial remineralizante (RE) preparada com 1,5 $\left.\mathrm{mmol} / \mathrm{L} \mathrm{Ca}^{+2}, 0,9 \mathrm{mmol} / \mathrm{L}_{\left(\mathrm{PO}^{-3}\right.}\right)_{4}, 0,15 \mathrm{~mol} / \mathrm{L} \mathrm{KCL}$ e tampão de cacodilato de $20 \mathrm{mmol} / \mathrm{L}$ em pH 7.0.

Para a realização da ciclagem, os corpos-de-prova foram mantidos imersos durante 6 horas em $15 \mathrm{~mL}$ de solução de saliva artificial desmineralizante a fim de assegurar a ação do $\mathrm{pH} 4,3$, seguindo-se de três lavagem consecutivas com água deionizada. A seguir os corpos-de-prova foram mantidos em $15 \mathrm{~mL}$ de solução de saliva artificial remineralizante com o objetivo de assegurar o $\mathrm{pH} 7,0$ durante 17 horas; de modo semelhante seguiram-se três lavagem consecutivas com água deionizada. A ciclagem foi realizada diariamente no mesmo horário e os espécimes foram conservados em estufa a $37^{\circ} \mathrm{C}$ durante todo o tempo de imersão em cada solução. Essa fase da pesquisa durou 14 dias, com descanso, em solução remineralizante, de 48 horas a cada 5 dias $^{11,12}$.

A mensuração em miligramas de cada um dos 60 corpos-de-prova em triplicata foi determinada antes e após a ciclagem, utilizando-se a balança analítica de precisão Gehaka Indústria e Comercio, Eletroeletrônicos Ltda., modelo AG 200.

\section{AVALIAÇÃO DE CARACTERÍSTICAS VISUAIS}

Para a avaliação da imagem visual do fio ortodôntico foram considerados os critérios de lisura, opacidade, brilho, ranhura e fissura, sendo realizada por um único observador, devidamente autocalibrado, utilizando como instrumento a Lupa Estereomicroscópio de Luz Opton, modelo TNE - 10b binocular. A opacidade e o brilho foram avaliados de forma conjunta, enquanto que para a análise das fissuras foram considerados um "corte/ rachadura" maior que as ranhuras, enquanto que estas foram consideradas como pequenos arranhões que não seguiam as projeções inerentes aos fios ortodônticos. 0 aumento utilizado nesse experimento foi de 1,5 vezes, sendo analisada toda a superfície do fio, enquanto que as imagens foram obtidas na parte central do espécime.

\section{RESULTADOS}

Os resultados foram analisados a partir da aplicação dos critérios de massa e das características visuais. A análise da perda de massa demonstrou que não houve diferença significativa entre o tempo inicial e após a ação do pH 4.3 independente da constituição dos fios e dos fabricantes. (Tabela 2). O GT5 foi o que apresentou a menor variação de massa, uma vez que a diferença percentual entre as médias do tempo inicial e do tempo final dos dez corpos-de-prova avaliados, foi da ordem de $0,042039855 \%$. Por outro lado, o grupo GT2 foi o que apresentou a maior variação de massa, exibindo como média dos dez corpos-de-prova 0,458236763\%, o que corresponde à diferença percentual entre o tempo inicial e o final. No entanto, ambos os grupos, obtiveram, aparentemente, ganho de massa ainda de acordo com os dados da Tabela 2, não obstante ter sido acordado que não houve alteração de massa significativa em todos os grupos analisados

Tabela 2 - Média inicial e final da massa em função do tempo; e diferença percentual entre a massa inicial e final

\begin{tabular}{l|r|r|r|r|r|r|r|r|r|r}
\hline & \multicolumn{1}{c|}{ Cp1 } & \multicolumn{1}{c|}{ Cp2 } & \multicolumn{1}{c|}{ Cp3 } & \multicolumn{1}{c|}{ Cp4 } & \multicolumn{1}{c|}{ Cp5 } & \multicolumn{1}{c|}{ Cp6 } & Cp7 & \multicolumn{1}{c|}{ Cp8 } & Cp9 & Cp10 \\
\hline GT1mi & 42,133 & 41,667 & 41,267 & 41,867 & 41,767 & 39,567 & 42,667 & 42,533 & 42,5 & 41,7 \\
\hline GT1mf & 42,067 & 41,267 & 41 & 41,367 & 41,967 & 39,833 & 42,867 & 42,333 & 42,067 & 42,2 \\
\hline GT1df & $-0,15821$ & $-0,96$ & $-0,64621$ & $-1,19427$ & 0,478851 & 0,673951 & 0,46875 & $-0,47022$ & $-1,0196$ & 1,199041 \\
\hline GT2mi & 34,833 & 34,4 & 35,467 & 34,433 & 34,067 & 34,867 & 35,133 & 34,633 & 35,4 & 35,933 \\
\hline GT2mf & 35,233 & 34,833 & 35,367 & 34,867 & 33,967 & 35,733 & 35,033 & 34,867 & 35,4 & 35,467 \\
\hline GT2df & 1,148325 & 1,25968 & $-0,28195$ & 1,25849 & $-0,29354$ & 2,48564 & $-0,28463$ & 0,673744 & 0 & $-1,29868$ \\
\hline GT3mi & 39,233 & 39,333 & 39,767 & 39,267 & 39,6 & 41,967 & 40,933 & 39 & 39,867 & 41,233 \\
\hline GT3mf & 39,733 & 39,467 & 38,933 & 39,667 & 40,333 & 41,867 & 40,633 & 38,933 & 39,6 & 41,333 \\
\hline GT3df & 1,274427 & 0,339 & $-2,09557$ & 1,018676 & 1,851843 & $-0,23828$ & $-0,7329$ & $-0,17095$ & $-0,6689$ & 1,274427 \\
\hline GT4mi & 33,9 & 33,9 & 34,033 & 33,867 & 33,667 & 33,867 & 32,767 & 34,467 & 34,133 & 34,067 \\
\hline GT4mf & 33,367 & 33,833 & 33,933 & 34,067 & 34,333 & 33,8 & 33,2 & 34,333 & 33,833 & 34,167 \\
\hline GT4df & $-1,57324$ & $-0,19667$ & $-0,29383$ & 0,590551 & 1,980178 & $-0,19686$ & 1,322472 & $-0,38687$ & $-0,87891$ & 0,293542 \\
\hline GT5mi & 39,6 & 40,233 & 39,333 & 38,8 & 41,667 & 40,6 & 35,867 & 40,567 & 40,7 & 39,067 \\
\hline GT5mf & 39,9 & 40,233 & 39,333 & 38,9 & 41,467 & 40,067 & 35,4 & 40,867 & 41,4 & 39,033 \\
\hline GT5df & 0,757576 & 0 & 0 & 0,257732 & $-0,48$ & $-1,31362$ & $-1,30112$ & 0,739523 & 1,719902 & $-0,08534$ \\
\hline GT6mi & 34,633 & 33,667 & 33,167 & 32,7 & 34,267 & 33,233 & 33,5 & 32,433 & 32,533 & 33,767 \\
\hline GT6mf & 35,367 & 33,333 & 33,133 & 32,033 & 34,067 & 33,033 & 33,8 & 32,9 & 32,633 & 32,967 \\
\hline GT6df & 2,11744 & $-0,99012$ & $-0,10052$ & $-2,03875$ & $-0,58366$ & $-0,60181$ & 0,895522 & 1,438859 & 0,307377 & $-2,3692$ \\
\hline
\end{tabular}

$G T=$ Grupo Teste; $m i=$ média inicial (antes da ciclagem); $m f=$ média final depois da ciclagem);

$d f=$ Diferença percentual em relação à massa inicial e final: fórmula [(Mfx100)/Mi] -100

Fonte Dados da pesquisa 
No tocante às características visuais, conforme os dados explicitados na Tabela 3, a análise dos corpos-de-prova frente à comparação das imagens nos tempos inicial e final demonstrou que os grupos GT1, GT3 e GT5 constituídos da liga do tipo $\mathrm{CrNi}$ - aço 302, mantiveram a mesma lisura em cinco espécimes no total dos dez que possuíam essa característica inicial. Por outro lado, os grupos GT2, GT4 e GT6 formados pela liga NiTi mantiveram ou pode-se admitir que melhoraram a característica de lisura. Entre os corpos-de-prova do grupo GT2, oito mantiveram a mesma lisura apresentada inicialmente, sendo que nos dois espécimes restantes foi observado ter melhorado essa característica. Quanto aos do grupo GT4, seis mantiveram a mesma lisura após da ciclagem, porém, em dois corpos-de-prova foi visualizada uma redução da lisura, enquanto que nos outros dois restantes constatou-se ter melhorado essa qualidade após a ciclagem. Portanto, o grupo GT4 apresentou uma lisura satisfatória em oito corpos-de-prova tanto no momento inicial quanto no final da experimentação.

Tabela 3 - Porcentagem dos grupos nas fases inicial e final de acordo com as características visuais.

\begin{tabular}{l|ccccc}
\hline $\begin{array}{c}\text { GRUPO/ } \\
\text { FASE }\end{array}$ & \multicolumn{5}{|c}{ CARACTERISTICAS VISUAIS } \\
\hline & LISURA & OPACIDADE & BRILHO & FISSURAS & RANHURAS \\
GT1 INICIAL & $100 \%$ & $90 \%$ & $70 \%$ & $50 \%$ & $70 \%$ \\
GT1 FINAL & $50 \%$ & $70 \%$ & $80 \%$ & $40 \%$ & $60 \%$ \\
GT2 INICIAL & $80 \%$ & $60 \%$ & $100 \%$ & $20 \%$ & $50 \%$ \\
GT2 FINAL & $100 \%$ & $100 \%$ & $90 \%$ & $10 \%$ & $20 \%$ \\
GT3 INICIAL & $100 \%$ & $80 \%$ & $70 \%$ & $20 \%$ & $10 \%$ \\
GT3 FINAL & $50 \%$ & $90 \%$ & $60 \%$ & $20 \%$ & $30 \%$ \\
GT4 INICIAL & $80 \%$ & $90 \%$ & $80 \%$ & $10 \%$ & $80 \%$ \\
GT4 FINAL & $80 \%$ & $70 \%$ & $80 \%$ & $20 \%$ & $60 \%$ \\
GT5 INICIAL & $100 \%$ & $100 \%$ & $60 \%$ & $20 \%$ & $20 \%$ \\
GT5 FINAL & $50 \%$ & $80 \%$ & $90 \%$ & $30 \%$ & $0 \%$ \\
GT6 INICIAL & $90 \%$ & $70 \%$ & $100 \%$ & $10 \%$ & $40 \%$ \\
GT6 FINAL & $90 \%$ & $90 \%$ & $100 \%$ & $20 \%$ & $60 \%$ \\
\hline AT & $90 \%$ &
\end{tabular}

A porcentagem indica o número de corpos de provas correspondente ao total por grupo $(n=10)$ que conseguiu manter ou melhorar aquela característica visual

Fonte Dados da pesquisa

De modo semelhante, no grupo GT6 oito corpos-de-prova mantiveram a mesma lisura após a ciclagem, sendo observado, entretanto, que um dos corpos-de-prova deste grupo apresentou comprometimento, ao passo que em outro a lisura foi melhorada após a ciclagem. Dessa forma, no tempo inicial nove corpos-de-prova apresentaram lisura adequada e apesar da influência ocorrida pela ciclagem no tempo final mantiveram esta característica satisfatória.

Com relação ao aspecto opacidade, foi constatada uma variação bem reduzida quando comparados os corpos-de-prova entre si no momento inicial e no momento final, independente do grupo avaliado, à exceção do grupo GT2 em que inicialmente apenas seis espécimes exibiam opacidade, após a ciclagem todos os dez corpos-de-prova passaram a apresentar essa característica no final da experimentação.

No tocante à propriedade brilho, os espécimes dos grupos GT1, GT3 e GT5 constituídos pela liga $\mathrm{NiCr}$ - aço 302 , foram os que apresentaram o menor brilho, tanto no instante inicial quanto no segundo tempo. Em contrapartida, os corpos-de-prova dos grupos GT2, GT4 e GT6 formados pela liga $\mathrm{NiTi}$, foram os que contiveram o maior número de espécimes exibindo brilho.

$\mathrm{Na}$ avaliação da possibilidade de fissura foi constatado que em cada grupo o número máximo de corpos-de-prova que apresentou essa característica no momento inicial e no final foi cinco.

A seguir, as figuras dos Grupos testes 1, 5 e 6, inicial e final, demonstram parcela das imagens estereoscópicas obtidas.

Figura 1 - Imagens dos fios ortodônticos antes e após a ciclagem

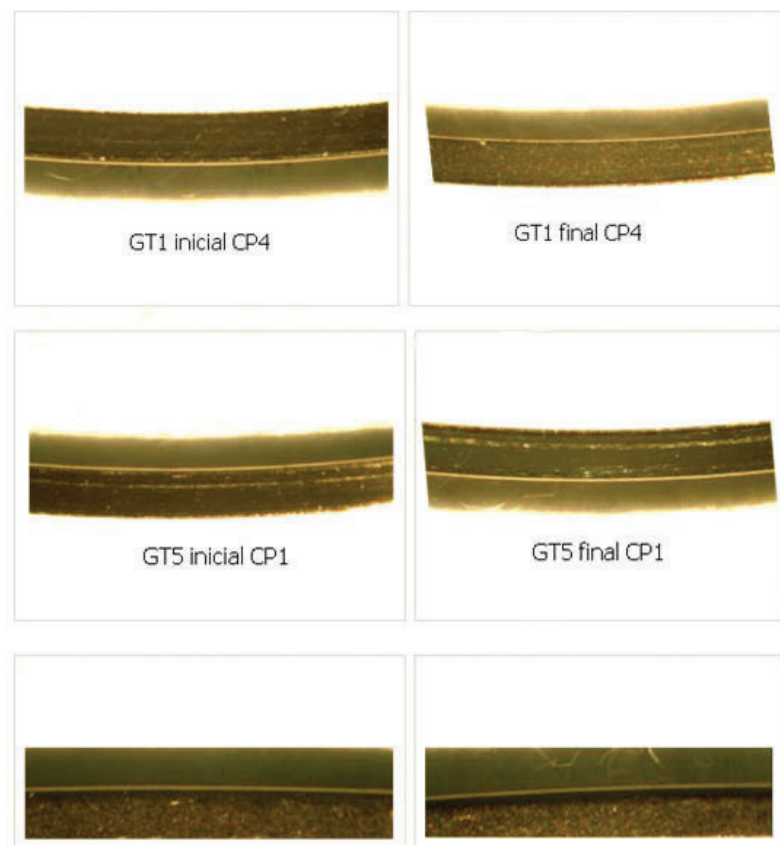

GT6 inicial CP5

GT6 final CP5

Fonte Autoria própria

\section{DISCUSSÃO}

O presente estudo demonstrou que os fios ortodônticos submetidos à ciclagem de $\mathrm{pH}$ no limite 4.3 , portanto abaixo do valor crítico, não foi capaz de promover uma perda de massa significativa ao ponto de proporcionar alterações nas características visuais constatadas estereoscopicamente. 
A determinação da perda de massa de corpos-de-prova após a ciclagem de $\mathrm{pH}$, de acordo com o protocolo experimental estabelecido, pode servir como fundamento para demonstrar perdas estruturais significativas. Os resultados do presente estudo demonstraram que não houve perda de massa significativa dos fios ortodônticos testados após quinze dias de incubação em saliva artificial - pH 7.0, previamente submetidos experimentalmente, ao pH 4.3. Corroborando com esta informação, Tahmasbi, Sheikh e Hemmati ${ }^{5}$ num artigo com metodologia similar apresentaram resultados próximos, não demonstrando perda de massa significativa entre os grupos ${ }^{5}$. Não obstante, em estudo anterior, Tahmasbi, Ghorbani e Masudrad utilizando um enxaguatório bucal contendo flúor terem observado que os íons de cobre liberados das amostras com fio de NiTi e braquetes foram maiores do que os das amostras contendo fio SS 4 .

Em que pese a literatura que trata das características visuais ser limitada, assim como a superfície dos fios ortodônticos comercializados serem considerados intactos, nem sempre estão em perfeitas condições físicas quando visualizados em microscopia, muito embora seja difícil visualizar pequenas corrosões, apesar do recurso experimental de ciclagem de $\mathrm{pH}$, se julgados apenas, por suas características visuais ${ }^{13}$.

Esse resultado da liga NiTi pode ser explicado pela combinação do Ti contido na liga metálica e o $\mathrm{O}_{2}$ formando o $\mathrm{TiO}_{2}$ que confere um melhoramento das suas propriedades dentre as quais a resistência a corrosão ${ }^{16-18}$. Outros estudos também demonstraram a formação de uma camada passiva na liga de aço inoxidável $\mathrm{CrNi}$ - 302. Isso pode ser explicado pela combinação de $\mathrm{Fe}-\mathrm{Cr}-\mathrm{Ni}$ mediante a formação de um fino filme conhecido como camada passiva. Assim sendo, esta película transparente e continua se forma na superfície do aço pela combinação do cromo presente no aço com o oxigênio do ar protegendo a superfície ${ }^{14,15}$.

Pesquisas demonstram a possibilidade de existência de rugosidade na superfície dos fios ortodônticos, logo, a lisura é um elemento que deve ser considerado em virtude da tendência à corrosão ${ }^{11}$. Desse modo a liga do tipo NiTi embora apresente tendência à corrosão, possuindo uma boa lisura no instante inicial, consegue manter a qualidade apesar da ciclagem ácida, divergindo do estudo que afirmava que a camada do NiTi é menos estável do que as ligas padrão ${ }^{19}$.

\section{CONCLUSÃO}

$\mathrm{Na}$ avaliação geral das características visuais, quando comparamos os grupos testes com seus respectivos grupos controle não houve alteração significativa. No entanto, quando analisado separadamente o grupo teste foi observado dentro desse grupo resultados divergentes assim como quando analisado o grupo controle separadamente o qual também apresentou resultado distintos. Essa diferença pode ser explicada porque cada tipo de liga possui suas peculiaridades devido aos diferentes processos de fabricação que são submetidas.

\section{REFERÊNCIAS}

KAROV, J.; HINBERG, I. Galvanic corrosion of selected dental alloys. J. Oral Rehabil., Oxford, v.28, n.3, p. 212-219, 2001.

SARANYA, R. et al. Corrosion resistance of metals and alloys in artificial saliva - an overview. Eur Chem Bull, [s.I], v.2, n.4, p.163-170, 2013.

HERNÁNDEZ CASTAÑEDA, A.; ARANZAZU MOYA, G. Características y propiedades físico-químicas de la saliva: una revisión. UstaSalud, [s.I], v.11, n.2, p.102, 2012.

TAHMASBI, S.; GHORBANI, M.; MASUDRAD M. Galvanic Corrosion of and Ion Release from Various Orthodontic Brackets and Wires in a Fluorideco,ntaining Mouthwash. J. Dent. Res. Dent. Clin. Dent. Prospects, Iran, v.9, n.3, p.159-165, 2015.

TAHMASBI, S.; SHEIKH, T.; HEMMATI, Y. Ion Release and Galvanic Corrosion of Different Orthodontic Brackets and Wires in Artificial Saliva. J. Contemp. Dent. Pract.. India, v.18, n.3, p.222-227, 2017.

MERÇON, F.; GUIMARÃES, P.; MAINIER, F. Corrosão: um exemplo usual de fenômeno químico. 19 ed. [S.I]: Química nova na escola, 2004.

ANDREASEN, G.; HILLEMAN, T. An evaluation of 55 cobalt substituted nitinol wire for use in orthodontics. J. Am. Dent. Assoc., Chicago, v.82, n. 6, p.1373-1375, 1971.

ORTIZ, A. et al. Metallic ions released from stainless steel, nickel-free, and titanium orthodontic alloys: Toxicity and DNA damage. Am. J. Orthod. Dentofacial Orthop., St. Louis, v.140, n.3, p.e115-e122, 2011.

KIM, H.; JOHNSON, J. Corrosion of stainless steel, nickel-titanium, coated nickel-titanium, and titanium orthodontic wires. Angle Orthod, Appleton, v.69, n.1, p. 39-44, 1999.

QUINTÃO, C.C.A.; BRUNHARO, I.H.V.P. Fios ortodônticos: conhecer para otimizar a aplicação clínica. Rev. Dent. Press Ortod. Ortop. Facial, Maringa, v.14, n.6, p.144-157, 2009.

McINTYRE, J.; FEATHERSTONE, J.; FU, J. Studies of dental root surface caries. 1: comparison of natural and artificial root caries lesions. Aust. Dent. J., Sydney, v. 45, n.1, p. 24-30, 2000.

McCINTYRE, J.; FEATHERSTONE, J.; FU, J. Studies of dental root surface caries 2: the role of cementum in root surface caries. Aust. Dent. J., Sydney, v.45, n.2, p.97-102, 2000.

KAMEDA T, ODA H, OHKUMA K, TERADA K. Effects of magnetic fields from electric toothbrushes on fluoride - and oral bacteria-induced corrosion of orthodontic metallic wires. Dent. Mater. J., Tokyo, v.38, n. 6, p.909-920, 2019.

ASM, METALS HANDOOK. Corrosion. 9 ed. Park-Ohio: Materials, 1917. v.13. p. $1200-1315$.

POURBAIX, M. Lições de corrosão eletroquímica. 3 ed., Bruxelas: Cebelcor, 1987, p.1-5,250-360.

WIDU, F. et al. Journal of Materials Science: Materials in Medicine. v. 10, n.5, p. 275-228, 1999.

LEE, T. et al. Corrosion resistance of different nickel-titanium archwires in acidic fluoride-containing artificial saliva. Angle Orthod, Appleton, v.80, n.3, p.547-553, 2010

SUGISAWA, $\mathrm{H}$, et al. Corrosion resistance and mechanical properties of titanium nitride plating on orthodontic wires. Dent. Mater. J., Tokyo, v.37, n.2, p. 286-292, 2018.

SARKAR, N. et al. The chloride corrosion behaviour of four orthodontic wires J. Oral Rehabil., Oxford, v.10, n.2, p. 121-128, 1983.

Submetido em: 09/11/2020

Aceito em: $12 / 11 / 2020$ 